\title{
SEMIGROUPS WITH POSITIVE DEFINITE STRUCTURE
}

\author{
PARFENY P. SAWOROTNOW
}

\begin{abstract}
Let $G$ be a semigroup with the identity 1 and an involution $x \rightarrow x^{*}$. One can define a complex-valued and an $H^{*}$. algebra valued positive definite function on $G$ in the obvious way. Assume that for each $x \in G$ there exists a positive number $L_{x}$ such that $q\left(x^{*} x\right) \leqq L_{x} q(1)$ for each complex positive definite function on $G$. It is shown that each $H^{*}$-algebra valued positive definite function on $G$ is of the form $p(x)=\left(f, T_{x} f\right)$ for some representation $T$ of $G$ on a Hilbert module $H$ and $f \in H$. Also there is an analogue of Bochner theorem for $G$.
\end{abstract}

1. In making a comparison between representation theories of groups and algebras one can observe certain similarities between these two theories. In both cases there is a correspondence between representations and certain types of positive definite functions [8], [10]. Also there is an analogue of Bochner theorem [9], [11] in each case. This observation suggests that there is a more general unifying theory which generalizes both the theory of representations of groups and the theory of representations of algebras.

The intent of this note is to present theories of this kind.

2. Let $G$ be a semigroup with identity 1 and an involution $x \rightarrow x^{*}$ (it has the property that $1^{*}=1$ and $x^{* *}=x,(x y)^{*}=y^{*} x^{*}$ for all $\left.x, y \in G\right)$. A complex-valued function $q$ on $G$ is said to be positive definite if $\sum_{i, j} \bar{\lambda}_{i} \lambda_{j} q\left(x_{i}^{*} x_{j}\right) \geqq 0$ for each pair $\left\{\lambda_{1}, \lambda_{2}, \cdots, \lambda_{n}\right\},\left\{x_{1}, \cdots, x_{n}\right\}$ of finite sets of complex-numbers and members of $G$ respectively. It is strictly positive definite if the equality holds only when $\lambda_{1}=\lambda_{2}=\cdots=\lambda_{n}=0$.

Definition 1. Assume that the family $Q$ of complex positive definite functions on $G$ is not empty. Then $G$ is said to have a positive definite structure if for each $x \in G$ there exists a positive number $L_{x}$ such that $q\left(x^{*} x\right) \leqq L_{x} q(1)$ for all $q \in Q$ (note that $q\left(x^{*} x\right) \geqq 0$ ). The ordered pair $(G, Q)$ will be called "semigroup with positive definite structure".

EXAMPLE 1. Let $G$ be an abstract group (or a topological group) such that the set $Q$ of positive definite functions on $G$ is not empty (different examples of such groups can be found on pp. 280-281 of [2]). Then

Presented to the Society, September 1, 1972; received by the editors July 17, 1972 and, in revised form, January 27, 1973.

AMS (MOS) subject classifications (1970). Primary 43A65, 42A88; Secondary 43A35, 46K15, 46G10, 28A45, 60G10.

c) American Mathematical Society 1973 
$(G, Q)$ is a semigroup with positive definite structure if we set $x^{*}=x^{-1}$ (in this case $q\left(x^{*} x\right)=q(1)$ for all $x \in G$ ).

Now let $A$ be a proper $H^{*}$-algebra [1] and let $\tau(A)=\{x y \mid x, y \in A\}$ be its trace-class [12]. A mapping $p: G \rightarrow \tau(A)$ is called an $A$-valued positive definite function on $G$ if $\sum_{i, j} a_{i}^{*} p\left(x_{i}^{*} x_{j}\right) a_{j} \geqq 0$ for all subsets $\left\{a_{1}, a_{2}, \cdots, a_{n}\right\}$ of $A$ and $\left\{x_{1}, \cdots, x_{n}\right\} \subset G$. As in the complex case, $p$ is strictly positive definite if $\sum_{i, j} a_{i}^{*} p\left(x_{i}^{*} x_{j}\right) a_{j}>0$ except for the trivial case.

REMARK. Above we are assuming that the range of the function $p$ is a subset of $\tau(A)$. This assumption is needed in the proof of Theorem 1 below to verify that $p(x)=\left(f_{0}, T_{x} f_{0}\right)$ (see [8, pp. 147-148]). However it is possible that this assumption could be derived from the other properties of the positive definite function $p$ and we need only to assume that $p(x) \in A$ for each $x$. We leave this as an open problem.

We define a *-representation of $G$ to be a mapping $x \rightarrow T_{x}$ of $G$ into bounded $A$-linear operators on a Hilbert module such that $T_{1}=I$, $T_{x^{*}}=T_{x}^{*}$ and $T_{x y}=T_{x} T_{y}$ for all $x, y \in G$. The next theorem generalizes Theorem 1 in [8] as well as Theorem 1 in $\S 30$ of [6].

THEOREM 1. Let $(G, Q)$ be a semigroup with positive definite structure. Then for each A-valued positive definite function $p$ on $G$ there exists a Hilbert module $H, a{ }^{*}$-representation $x \rightarrow T_{x}$ by bounded A-linear operators on $H$ and $f_{0} \in H$ such that $p(x)=\left(f_{0}, T_{x} f_{0}\right)$ and $\left\|T_{x}\right\|^{2} \leqq L_{x}$ for all $x \in G$. If $p$ is strictly positive definite then the mapping $x \rightarrow T_{x}$ is an isomorphism.

The corresponding theorem holds for complex-valued positive definite functions.

Proof. Fix $a \in A$ and consider the function $q(x)=\operatorname{tr}\left(a^{*} p(x) a\right)$. Then $q \in Q$ and as in [6, §30] one can show that $q\left(x^{*}\right)=\bar{q}(x)$ for each $x \in G$ : if $\lambda$ is any complex number then $q(1)+\lambda q(x)+\bar{\lambda} q\left(x^{*}\right)+|\lambda|^{2} q\left(x^{*} x\right)$ is real; this means that $\alpha=q(x)+q\left(x^{*}\right)$ and $\beta=i q(x)-i q\left(x^{*}\right)$ are real and so $\bar{q}(x)=(\alpha+i \beta)^{-}=\alpha-i \beta=q\left(x^{*}\right)$. Now, using the principal argument in the proof of Lemma 1 of $[10]$, one can show that $p\left(x^{*}\right)=p(x)^{*}$ for all $x \in G$. After that we can proceed as in the proof of Theorem 1 of [10]. Let $K$ be the set of all formal finite sums $f=\sum_{i} x_{i} a_{i}, g=\sum_{j} y_{j} b_{j}$ with $x_{i}, y_{j} \in G$ and $a_{i}, b_{j} \in A$. Define $(f, g)=\sum_{i, j} a_{i}^{*} p\left(x_{i}^{*} y_{j}\right) b_{j}$, consider

$$
\mathfrak{N}=\{f \in K \mid(f, f)=0\}
$$

and let $H$ be the completion of the space $H^{\prime}=K / \mathfrak{N}$ with respect to the norm $\|f\|=(\operatorname{tr}(f, f))^{1 / 2}$ (if $p$ is strictly positive definite then $\mathfrak{N}=(0)$ and $\left.H^{\prime}=K\right)$. Now for each $x \in G$ define $T_{x}(f)=T_{x}\left(\sum x_{i} a_{i}\right)=\sum x x_{i} a_{i}$. The condition " $q\left(x^{*} x\right) \leqq L_{x} q(1), q \in Q$ " implies that $T_{x}$ will induce a bounded linear operator on $H$ (which we will also denote by $T_{x}$ ): let $f=\sum x_{i} a_{i}$ be fixed; then $q_{0}(x)=\operatorname{tr}\left(f, T_{x} f\right)=\operatorname{tr}\left(\sum_{i j} a_{i}^{*} p\left(x_{i}^{*} x x_{j}\right) a_{j}\right)$ is a member of 
$Q$, which means that $q_{0}\left(x^{*} x\right) \leqq L_{x} q_{0}(1)$ (and this can be rewritten as " $\left\|T_{x} f\right\|^{2} \leqq L_{x}\|f\|^{2}$ "). It follows that $T_{x}$ is a bounded ( $A$-linear) operator on $H$ and $\left\|T_{x}\right\|^{2} \leqq L_{x}$ for each $x \in G$.

Also as in [8, pp. 147-148] one can show that there exists $f_{0} \in H$ such that $p(x)=\left(f_{0}, T_{x} f_{0}\right)$ for all $x \in G$. If $q$ is strictly positive definite then the mapping $x \rightarrow T_{x}$ is an isomorphism: if $x \neq y$ then $0<p\left(x^{*} x\right)-p\left(x^{*} y\right)-$ $p\left(y^{*} x\right)+p\left(y^{*} y\right)=\left\|\left(T_{x}-T_{y}\right) f_{0}\right\|^{2}$ and so $T_{x} \neq T_{y}$.

REMARK. Lindahl and Maserick [3, Theorem 3.2] have a similar result but they only consider complex positive definite functions.

3. Now let us assume that $G$ is commutative. Then we have the following abstract analogue of Bochner theorem (compare with [9] and [11]). (Note that, unlike Lindahl and Maserick [3, Theorem 2.1], we do not assume the function $p$ below to be bounded.)

THEOREM 2. Let $G$ be a commutative semigroup having a positive definite structure. Then for each A-valued positive definite function $p$ on $G$ there exists a compact Hausdorff space $\mathfrak{M}$, a homomorphism $x \rightarrow x($ ) of $G$ into a multiplicative semigroup of complex-valued continuous functions on $\mathfrak{M}$ and a positive A-valued measure $\mu$, defined on Borel subsets of $\mathfrak{M}$, such that $p(x)=\int_{\mathfrak{M}} x(M) d \mu(M)$ for each $x \in G$. If $p$ is strictly positive definite then the mapping $x \rightarrow x()$ is an isomorphism.

The corresponding theorem holds for a complex-valued positive definite function.

Proof. Let $x \rightarrow T_{x}$ be a ${ }^{*}$-representation of $G$ on a Hilbert module $H$ such that $p(x)=\left(f_{0}, T_{x} f_{0}\right)$ for some $f_{0} \in H$ (use Theorem 1 above). Let $B$ be the $B^{*}$-algebra generated by the operators $T_{x}, x \in G$. Then $B$ is commutative and so there exists a compact Hausdorff space $\mathfrak{M}$ such that $B$ is isomorphic and isometric to the algebra $C(\mathfrak{M})$ of all continuous complexvalued functions on $\mathfrak{M}[\mathbf{4}$, p. 88].

For a given $x \in G$ let $x()$ be the member of $C(\mathfrak{M})$ corresponding to the operator $T_{x}$. Then it follows from Proposition II of subsection 4 of $\S 17$ of [6] that there exists a spectral measure $P: \Delta \rightarrow P_{\Delta}$ defined on Borel subsets of $\mathfrak{M}$, such that $T_{x}=\int_{\mathfrak{M}} x(M) d P_{M I}$ for all $x \in G$. As in [11] we conclude that each $P_{\Delta}$ is $A$-linear [7] (we use the fact that each $P_{\Delta}$ commutes with each bounded linear operator that commutes with all $T_{x}$, $x \in G)$; so $P$ is a generalized spectral measure $[9$, p. 118].

Now we define the measure $\mu$ by setting $\mu(\Delta)=\left(f_{0}, P_{\Delta} f_{0}\right)$ and verify:

$$
\begin{aligned}
p(x) & =\left(f_{0}, T_{x} f_{0}\right)=\left(f_{0},\left(\int x(M) d P_{M}\right) f_{0}\right) \\
& =\int x(M) d\left(f_{0}, P_{M} f_{0}\right)=\int x(M) d \mu(M)
\end{aligned}
$$

(see Lemma 1 in [9]). 
4. In this section we shall present a more general theory which includes the above theorems as well as theorems about representations of a Banach *-algebra [10], [11] as special cases.

Definition 2. Let $G$ and $A$ be as above, and let $p$ be an $A$-valued positive definite function on $G$. A derivate of $p$ is a function on $G$ of the form $p^{\prime}(x)=\sum_{i, j} a_{i}^{*} p\left(x_{j}^{*} x x_{j}\right) a_{j}$ for some $\left\{a_{1}, \cdots, a_{n}\right\} \subset A$ and some $\left\{x_{1}, \cdots, x_{n}\right\} \subset G$. A derivate of a complex positive definite function is a function $q^{\prime}(x)=\sum_{i, j} \bar{\lambda}_{i} \lambda_{j} q\left(x_{i}^{*} x x_{j}\right)$, where $\lambda_{1}, \cdots, \lambda_{n}$ are complex numbers. Note that derivates are also positive definite.

Definition 3. An $A$-valued (complex-valued) positive definite function $p$ is said to be representable if for each $x \in G$ there exists a positive number $L_{x}(p)$ such that $\tau\left(p^{\prime}\left(x^{*} x\right)\right) \leqq L_{x}(p) \tau\left(p^{\prime}(1)\right)\left(p^{\prime}\left(x^{*} x\right) \leqq L_{x}(p) p^{\prime}(1)\right)$ for each derivate $p^{\prime}$ of $p$.

Note that a semigroup $G$ with positive definite structure has the property that each positive definite function on $G$ is representable. In a more general case we may say that the semigroup has a partial positive definite structure.

EXAmple 2. Let $B$ be a Banach *-algebra with identity such that $\left\|x^{*}\right\|=$ $\|x\|$ for all $x \in B$. Then $[6, \S 10]$ each positive linear functional $q$ on $B$ satisfies the condition $q\left(x^{*} x\right) \leqq\left\|x^{*} x\right\| q(1)$, and this implies that each positive linear functional $p$ is a representable positive definite function with $L_{x}(p)=\left\|x^{*} x\right\|$. If $B=L^{1}(G) e[10$, p. 303] then each linear $A$-valued positive definite function on $B$ (positive $A$-functional in the terminology of [10]) is representable.

EXAMPLE 3. Let $G$ be a multiplicative semigroup of $A$-linear [7, p. 194] operators, acting on a Hilbert module $H$, closed under the involution $T \rightarrow T^{*}$ and including the identity operator $I$. If $f \in H$ then $p(T)=(f, T f)$ is a representable positive definite function on $G$.

THEOREM 3. For each representable A-valued positive definite function $p$ on a semigroup $G$ as above, there exists a Hilbert module $H, a *$-representation $x \rightarrow T x$ of $G$ on $H$ and $f \in H$ such that $p(x)=(f, T x f)$ and $\|T x\|^{2} \leqq$ $L_{x}(p)$ for all $x \in G$.

If $G$ is communicative then $p$ has a representation $p(x)=\int_{\mathfrak{B}} x(M) d \mu(M)$, where $\mathfrak{B}, \mu$ and $x(M)$ are as in Theorem 2 above.

We have also a corresponding theorem for complex-valued positive definite functions.

The proof of Theorem 3 is obvious in view of the proofs of Theorems 1 and 2 above.

5. We should like to mention here one of the applications of the above theory that may be of interest. Let $G$ be as above. One can define 
an abstract stationary process (stationary random field) as a mapping $\xi$ of $G$ into a Hilbert module $H$ such that $(\xi(t), \xi(s))=\left(\xi(1), \xi\left(t^{*} s\right)\right)$ for all $s, t \in G$. Then the first part of Theorem 3 implies that each representable positive definite function is of the form $p(t)=(\xi(1), \xi(t))$ for some abstract stationary process $\xi$ on $G$. This is a partial generalization of Theorem 5.1 in Chapter 1 in [14] and of Theorem 2 in [13].

The author would like to thank the referee for his critical comments.

\section{REFERENCES}

1. W. Ambrose, Structure theorems for a special class of Banach algebras, Trans. Amer. Math. Soc. 57 (1945), 364-386. MR 7, 126.

2. Edwin Hewitt and Kenneth A. Ross, Abstract harmonic analysis. Vol. II: Structure and analysis for compact groups analysis on locally compact Abelian groups, Die Grundlehren der math. Wissenschaften, Band 152, Springer-Verlag, New York and Berlin, 1970. MR 41 \#7378.

3. R. J. Lindahl and P. H. Maserick, Positive definite functions on involution semigroups, Duke Math. J. 38 (1971), 771-782.

4. L. H. Loomis, An introduction to abstract harmonic analysis, Van Nostrand, Princeton, N.J., 1953. MR 14, 883.

5. B. Sz.-Nagy, "Prolongements des transformations de l'espace de Hilbert qui sortent de cet espace," Appendice au livre Leçons d'analyse fonctionnelle par F. Riesz et B. Sz.Nagy, Akad. Kiadó, Budapest, 1955. MR 16, 837.

6. M. A. Naìmark, Normed rings, GITTL, Moscow, 1956; English transl., Noordhoff, Groningen, rev. ed., 1964. MR 19, 870; MR 34 \#4928.

7. P. P. Saworotnow, A generalized Hilbert space, Duke Math. J. 35 (1968), 191-197. MR 37 \#3333.

8. - Representation of a topological group on a Hilbert module, Duke Math. J. 37 (1970), 145-150. MR 41 \#7442.

9. - Bochner-Raikov theorem for a generalized positive definite function, Duke Math. J. 38 (1971), 117-121. MR 43 \#940.

10. - Generalized positive linear functionals on a Banach algebra with an involution, Proc. Amer. Math. Soc. 31 (1972), 299-304. MR 44 \#4528.

11. - Integral as a certain type of a positive definite function, Proc. Amer. Math. Soc. 35 (1972), 93-95.

12. P. P. Saworotnow and J. C. Friedell, Trace-class for an arbitrary $H^{*}$-algebra, Proc. Amer. Math. Soc. 26 (1970), 95-100. MR 42 \#2304.

13. P. P. Saworotnow, Abstract stationary processes, Proc. Amer. Math. Soc. 40 (1973), 583-587.

14. Ju. A. Rozanov, Stationary random processes, Fizmatgiz, Moscow, 1963; English transl., Holden-Day, San Francisco, Calif., 1967. MR 28 \#2580; MR 35 \#4985.

Department of Mathematics, Catholic University of America, Washington, D.C. 20017 